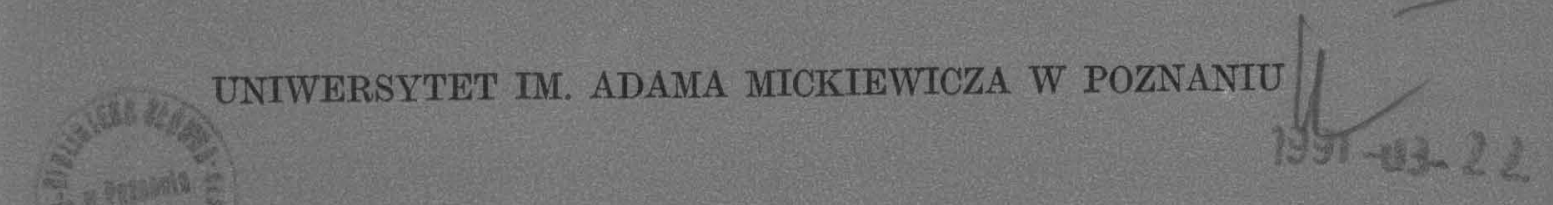

\title{
STUDIA GERMANICA POSNANIENSIA XIV
}

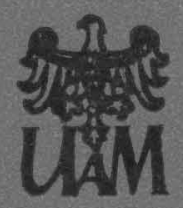

POZNAŃ 1990 



\section{STUDIA \\ GERMANICA POSNANIENSIA}

\section{XIV}

LITERATUR UND GESCHICHTSBEWUSSTSEIN

Herausgegeben von

A. Z. BZDĘGA, S. H. KASZYŃSKI, H. ORŁOWSKI

Sekretariat: CZ. KAROLAK

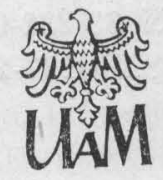

POZNAŃ 1990 

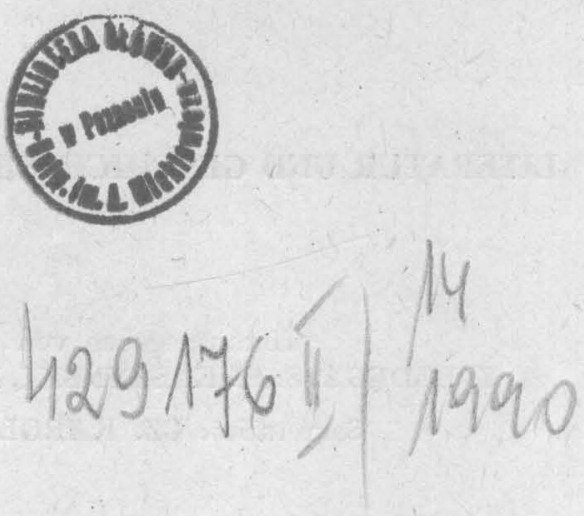

Redaktor: Anna Gierlińska

Redaktor techniczny: Michał Łyssowski

ISBN 83-232-0223-0

ISSN $0137-2467$

WYDAWNICTWO NAUKO WE UNIWERSYTETU IM. ADAMA MICKIEWICZA W POZNANIU

Nakład $380+80$ egz. Ark. wyd. 10,50. Ark. druk. 8,75. Papier druk. sat. kl. III. 80 g. $70 \times 100$. Oddano do skladania w lutym 1989 r. Podpisano do druku w pazdzierniku 1990 r. Druk ukonczono w listopadzie 1990 r. Zam. nr 198/142.

DRUKARNIA UNIWERSYTETU IM. ADAMA MICKIEW ICZA, POZNAN, UL. FREDRY 10

\section{Bibl. UAM}




\section{INHALT}

Edyta Połczyńska: Karl von Holteis Kościuszko-Verehrung . . . . . . . .

Jerzy Kałążny: Die Geschichtsauffassung von Alexander von Bronikowski. Zur Geschichte Polens und ihrer fiktionalen Komplementarität . . . . . . . Aleksandra $\mathrm{Lukomska-Woroch:} \mathrm{Das} \mathrm{historische} \mathrm{Prosawerk} \mathrm{von} \mathrm{Bruno} \mathrm{Frank}$ Detlef Ignasiak: Historisches Sujet und aktuelle Bezüge. Zu Bertold Brechts Erzählung Die Trophäen des Lukullus (1939) . . . . . . . . . . . . . . Roman Dziergwa: Heinrich Manns historischer Roman Henri Quatre in der polnischen Rezeption 1936-1985 ............... . Czeslaw Karolak: Deutsche Literatur nach dem Dritten Reich. Die fünfziger Jahre im ,toten Winkel" der Geschichte . . . . . . . . . . . . Hubert Orłowski: Zur Bedeutung Eichendorffs in den Romanen von Horst Bienek . . . . . . . . . . . . . . . . . . . .

Włodzimierz Bialik: Zwischen fragwürdiger Historiosophie und utopischen Entwicklungsperspektiven: Das weltanschauliche Angebot der beiden ersten Romane von Johannes Mario Simmel 


\title{
ZWISCHEN FRAGWÜRDIGER HISTORIOSOPHIE UND UTOPISCHEN ENTWICKLUNGSPERSPEKTIVEN: DAS WELTANSCHAULICHE ANGEBOT DER BEIDEN ERSTEN ROMANE VON JOHANNES MARIO STMMEL
}

\begin{abstract}
Włodzimierz Bialik, Zwischen fragwürdiger Historiosophie und utopischen Entwicklungsperspektiven: Das weltanschauliche Angebot der beiden ersten Romane von Johannes Mario Simmel [Between the problematic historiosophy and utopian perspectives of development: the world-view offer of two first novels of Johannes Mario Simmel], Studia Germanica Posnaniensia, Adam Mickiewicz University Press, Poznań, vol. XIV: 1990, pp. 119-138, ISBN 83-232-0223-0, ISSN 0137-2467.
\end{abstract}

In the article the author writes about two novels Mich wundert, daß ich so fröhlich bin (1948) and Das geheime Brot (1950). The problematic historiosophic conceptions which were at the basis of the first of the two novels by Simmel, and which pointed one time to the unmasking of the (false) historical consciousness of German society by the author, and another time to the unmasking of his (the author's) own problematic historical consciousness. These conceptions were "replaced" in the second novel additionally by the utopian naive conceptions of the further historical development of Germany. Early novels of Simmel are an evidence of world-view convulsions of the German society in the first post-war years and of helplessness of the author himself as far as the world-view.

Włodzimierz Bialik, Institute of German, Adam Mickiewicz University,Poznań-Poland.

Simmels erster Roman Mich wundert, daß ich so fröhlich bin (1948) wird von einem zweieinhalbseitigen Vorwort eröffnet, in dem die ganze Handlung von seiten des auktorialen, im nachhinein berichtenden Erzählers umrissen und einleitend kommentiert wird: Es ist März des Jahres 1945 in Wien. Infolge eines Luftangriffs werden in einem Keller im Zentrum der Stadt sieben einander bisher unbekannte Menschen verschüttet, die zufällig(?) gerade in diesem Keller Schutz vor amerikanischen Bomben suchten: das über sechzigjährige Fräulein Therese Reimann, Reinhold Gontard, der Priester einer nahegelegenen Kirche, die einunddreißigjährige hochschwangere Anna 
Wagner mit ihrer kleinen Tochter Evi, der in einem kri egswichtigen "Laboratorium arbeitende Chemiker Walter Schröder, das junge Mädchen Susanne Riemenschmied, die Schauspielerin werden wollte, und der fünfundzwanzigjährige desertierte Soldat Robert Faber.

Das simultan konstruierte erste Kapitel gibt den ersten Einblick in die Persönlichkeiten und Lebensläufe der später Verschütteten und zeichnet die letzten Minuten ihrer Wege, die sich dann im Keller des Hauses am Neuen Markt kreuzen sollen, auf die Weise ab, daß jedes Unterkapitel - ungeachtet kleiner Unterschiede - in derselben Zeit endet: mit dem Eintreffen des nächsten Schutzsuchenden.

Die drei Männer-Figuren stehen im Zentrum der geistigen Auseinandersetzung, die der Verschüttung folgt, für alle drei wurde der Krieg zum entscheidenden Erlebnis. Bei Reinhold Gontard, dem Geistlichen, hat er eine deutliche Spur hinterlassen: da er in Anbetracht der Allmacht der Greuel, die sich in Gestalt von Krieg der Welt bemächtigten, keine Möglichkeit der Theodizee sah, hörte er auf zu beten und fing an zu trinken. Er sah sich nicht imstande, den stummen Gott zu rechtfertigen oder ihm gar zu verzeihen: „Ein Gebet, das erst beantwortet wurde, wenn schon alles verloren und nicht wiedergutzumachendes Unheil geschehen war, entbehrte seines Sinnes und konnte ebenso unterbleiben. (...) Es kam ihm, gemäß seiner orthodoxen Erziehung, nicht in den Sinn, aufrührerisch mit seinem Herrn zu Gericht zu gehen, er bekümmerte sich bloß über ihn und fand, daß er ihm viel Schmerzen bereitete. Er grübelte, beklagte die Zeit und ihre unglücklichen Menschen und verlor seinen Glauben. Sonst tat er nichts"' (25).

Der Anorganiker Walter Schröder, ein überzeugter Nazi, bemerkte zwar ,in der Struktur und dem Wesen des Regimes, welchem er diente, Stellen, die ihm unrecht erschienen. Aber er nannte sie im stillen, extreme Schattenpunkte', wie sie überall auftreten, wo mit sehr hellem Licht hantiert wird, und erwartete, daß sie sich mit der Zeit neutralisieren und abschwächen würden” (37). Da der verlorene Krieg für ihn ,,das Ende seiner mühsam aufgebauten Existenz bedeuten würde", war er bereit, ,bedingungslos alles,was ihm von seiten der Parteikollegen mitgeteilt und eingeschärft wurde" zu glauben und ,,jederzeit ihre Anordnungen zu befolgen, so dumm, grenzenlos grausam und destruktiv sie auch sein mochten” (37). Schröder war ,ein Mann ohne Herz, den es leicht ankam, Unrecht zu tun, weil er an das Unrecht glaubte, weil er selbst ein Teil des Unrechts war" (37f).

Der letzte der Männer-Triade, Walter Faber, desertierte von der Front, nachdem er einen Offizier geschlagen und ihm die Pistole weggenommen hatte, als dieser einen ungarischen Jungen erschießen wollte. „Ich habe Menschen getötet" - sagt er - ,und den Mund gehalten, wenn Unrecht geschah. Sechs Jahre lang. Sechs Jahre lang habe ich den Mund gehalten zu viel schlimmeren Dingen als denen, die gestern geschahen. Ich habe ge- 
schwiegen und geschwiegen und geschwiegen. Und dann, gestern früh, auf einmal, da war es zu Ende. Und ich konnte nicht mehr zusehen. Und da lief ich fort, weil ich genug hatte. (...) Ich habe nicht versucht, einen der wirklich Schuldigen $\mathrm{zu}$ erledigen. Ich bin nur fortgelaufen, weil ich es nicht mehr aushalten konnte" (183).

In der für den Roman zentralen Krieg- und Faschismusdiskussion wird die Frage nach den Ursachen des Krieges in den Vordergrund gerückt, die Frage, die die handélnden Figuren zu beantworten versuchen. Schröder, der den Krieg als „Gesetz der Natur" ansieht, das es verhindert, daß die Übervölkerung „Katastrophen noch viel größeren Umfanges” (121) verursachen würde, gibt somit - entgegen vielen seiner Behauptungen - mittelbar zu, daß der Krieg doch ein Unheil ist. Er entwickelt eine absurde historiosophische Konzeption, nach der der Frieden in der Welt ein vorübergehender Zustand sein kann und der Krieg immer ,durch die Verbrechen anderer" (206) zustande kommt: ${ }^{1}$ „Es wäre lächerlich” - sagt er zu Gotard - „unter das Jahr 1933 einen Strich zu ziehen und zu sagen: an allem, was seither geschah, tragt ihr die Schuld. Wenn wir diesen Krieg verlieren, werden Sie noch sehen, daß ich recht habe. Denn dann wird es uns nicht besser gehen. Dann werden dieselben Greuel, deren man uns anklagt, von anderen begangen werden. Und wieder wird man jemanden suchen, der die Schuld an ihnen trägt. Man wird ihn finden. Und man wird vergessen, daß wir die Schuld an seiner Schuld tragen, so wie man schon vergessen hat, was vor uns geschah" (207). Auf diese Weise entsteht ein mörderisches Perpetuum mobile, das jeden nächsten Krieg verursacht und rechtfertigt.

Auffallend ist, daß - von einer einzigen unverständlichen Ausnahme abgesehen, von der hier noch die Rede sein wird - Schröder der Einzige ist, der hier - obwohl völlig falsch und von der Nazi-Ideologie vergiftet die Ursachen des Faschismus und als dessen Folge die des Krieges in äußeren Faktoren sieht, alle anderen suchen sie in den Menschen selbst: schuld sind sowohl die Befehlenden als auch die Ausführenden, schuldig sind also sowohl die Schreienden als auch die Schweigenden. Gontard versteht nicht, wie Schröder einverstanden sein kann mit dem, was er von seiner Führung hört, Schröder begreift nicht, wie Gontard schweigen kann, wenn er damit nicht einverstanden ist (209). In dem Menschen selbst, in seinem moralischen Versagen und seiner allumfassenden Angst wird das Böse gesucht, das den Krieg entfesselte. "Jeder von uns ist ein Schuft" - sagt Faber. ,In jedem von uns lebt die Gemeinheit. Sie ist sehr lebendig geworden. Denn wir haben sie gut genährt mit dem, was ihre Lieblingsspeise ist: mit unserer Angst" (188).

2 Als Verbrechen und Fehler anderer werden hier - was in diesem Kontext nicht verwundert - der erste Weltkrieg, der Vertrag von Versailles, "der eine Dummheit war" (206), sowio „das gewissenlose Betragen der Siegerstaaten" (207) genannt. 
Der ausgebliebene Widerstand gegen den Faschismus wird hier ,auf das Individuum und seine Charakterstruktur bzw. seine menschlich-allzumenschlichen Fehler" ${ }^{2}$ zurückgeführt. Heinz Brüggemann führt eine ganze Reihe von Textbeispielen an, die die obige These bezeugen, wenn er aber Simmel vorwirft, daß die Verselbständigung und Absolutheit der emotionellen und moralischen Faktoren ,bewußt die historische Erfahrung aus dem Bewußtsein verdrängt, daß solche Angst Millionen Menschen erst aufgezwungen werden konnte nach der Niederlage großer organisierter Bewegungen gegen den Faschismus" "3, so verkennt er zweierlei: erstens kann hier von irgendeiner bewußten Verdrängung nicht die Rede sein, weil es keine objektive Prämisse zu einer solchen Unterstellung gibt, und zweitens: Simmels Roman erhebt nicht den Anspruch, die objektiven Ursachen des Faschismus und des Krieges zu analysieren, sondern Meinungen der sog. gewöhnlichen Deutschen, die sich entweder von dem Faschismus verführen ließen oder nichts gegen ihn zu unternehmen imstande waren, zu konfrontieren. Die Weltanschauung der handelnden Figuren mit der des Autors (des Romans) zu identifizieren, bedeutet - obwohl die auktoriale Erzählsituation dazu provoziert $^{4}$ - eine unbegründete interpretatorische Fehldeutung. Simmels Roman ist nicht Auf-Klärung sondern Auf-Zeichnung: jeder seiner Helden präsentiert eine Meinung, keine von diesen Meinungen ist - in bezug auf die Ursachen des Faschismus und die Möglichkeiten, sich ihm zu widersetzen richtig, aber keine erhebt auch den Anspruch auf ausschließliche Richtigkeit. Das, was Simmel in seinem Roman zur Schau stellte, ist gerade die weltanschaulich-politische Unbeholfenheit der Deutschen nicht nur der Jahre 1933-1939, sondern auch 1939-1945, als es für viele schon klar wurde, was ,die Bewegung" wirklich mit sich bringt.

Es wäre zwar sehr wünschenswert - und daran lag es wohl auch Brügge-

2 H. Brüggemann, Johannes Mario Simmel - Deutsche Ideologie als Roman, in: H. L. A rnold (Hrsg.), Deutsche Bestseller - Deutsche Ideologie. Ansätze zu einer Verbraucherpolitik, Stuttgart 1975 , S. 81.

3 Ebenda, S. 82.

4 Dem Erzähler die Rolle des Trägers der Konzeption des Autors zu unterschieben, würde (und war schon mehrmals) eine grobe Vereinfachung und Grund verschiedenartiger Mißverständnisse bedeuten. „In früheren Arbeiten, die auf Erfahrungen des traditionellen auktorialen Romans fußten" - schreibt Stanisław Eile - ,schien das Problem einfach zu sein, weil man mit den Anschauungen des Autors nicht nur die Aussagen des Erzählers identifizierte, sondern die Meinung der Figuren, die nicht entweder im Kommentar oder durch die Selbstkompromittierung ihrer Träger abgelehnt wurden”. Die auktoriale Erzählsituation, in der der Erzähler aus der „Perspektive des Niemanden" berichtet und gegenüber der dargestellten Welt transzendent ist, wird noch heute oft mit der Erzählerperspektive des Schriftstellers identifiziert, ohne die - manchmal sehr wichtige und programmatische - Distanz zwischen dem Autor und seinem Erzähler zu beachtẹn. Vgl. dazu: S. Eile, Swiatopogląd powieści (Die Weltanschauung des Romans), Wrocław, Warszawa, Kraków, Gdańsk 1973, S, $7-24$ u. 45-52. 
mann - wenn einer der Helden behauptete: ,Als Ausdruck der allgemeinen Krise des Kapitalismus wird im Faschismus das Unvermögen der reaktionären Kräfte des Monopolkapitals sichtbar, die Volksmassen, vor allem die Revolutionäre Arbeiterbewegung, mit den Mitteln der bürgerlichen Demokratie zu beherrschen, sie den aggressiven imperialistischen Interessen unterzuordnen und die Macht des Monopolkapitals gegen die Kräfte des Fortschritts (...) aufrechtzuerhalten" 5 , aber wer sollte zu solchen Folgerungen kommen: der an Gott zweifelnde und aus Verzweiflung trinkende Priester, der einfache Soldat, eine der Frauen oder vielleicht der Nazi-Wissenschaftler? Es gibt nämlich im Roman keinen - und auch das ist symptomatisch - der, außer einzelnen triftigen Formulierungen ${ }^{6}$, eine Gesamtkonzeption oder wenigstens eine Gesamterklärung anbieten könnte. Nur der Priester, die widerspruchsvollste Figur der Handlung, rückt einmal - und das ist die schon erwähnte Ausnahme - in die Nähe der übrigens am allgemeinsten formulierten Wahrheit. Auf Schröders Frage, wie der Faschismus überhaupt zustande kam, antwortet er: „Es gibt dafür Gründe wirtschaftlicher und politischer Art” (206), um jedoch später Schröder ,das dumme Opfer einer Ereigniskette" zu nennen, ,die soweit zurückreicht, daß man sie nicht verfolgen kann" (295).

Brüggemanns Behauptung: ,Die fast vollständige Destruktion der historischen Kontinuität politischer Erfahrung und kollektiven, organisierten politischen Handelns, die dem Faschismus gelungen war, wird durch solchen hilflosen, moralischen Antifaschismus nur noch vertieft" ", trifft nicht zu: auch der moralische Antifaschismus braucht nicht hilflos zu sein, wenn die moralischen Erkenntnisse den Ausgangspunkt bilden würden für menschliche Aktivität, auch wenn sie - was Brüggemann als reaktionär bezeichnet ${ }^{8}$ auf den einzelnen Menschen bezogen wird. Diese menschliche Aktivität wird aber bei Simmel ununterbrochen in Frage gestellt und das - nicht sein ,moralischer Antifaschismus" - ist das Problematischste an seinem ersten Roman.

5 Meyers Neues Lexikon, Bd. 4, Leipzig 1972, S. 500.

- Solche Stellen kommen vor allem in Dialogpassagen vor, wo die Helden keine umfassenden Konzeptionen darlegen, sondern spontan ihre Reaktion auf das vorher Gesagte in Worte fassen. Ein Beispiel solcher Dialogführung sei hier genannt. Es sprechen Gontard und Schröder:

„Ein ganzes Volk hält den Mund zu dem, was die Führung sagt."

"Sie selber auch."

"Ich auch."

"Und Sie sollten nicht schwoigen!"

„Auch Sie nicht!"

"Ich bin einverstanden mit dem, was ich höre. Aber Sie sind es nicht."

„Ich begreife kaum", sagte der Priester, „wie Sie einverstanden sein können." „Und ich", erwiderte Schröder, „begreife nicht, wie Sie schweigen können."

7 H. Brüggemann, Johannes Mario Simmel..., a.a.O., S. 82.

8 Vgl: ebenda, S. 81. 
Der Erzähler läßt den Leser in seinem Vorwort wissen, daß er - bemüht, das Geheimnis der Verschütteten zu lüften - erst dann Frieden fand, „als er begriff, daß es nichts gibt auf dieser Welt, das ohne Grund und zufällig geschieht. Daß hinter allen Dingen ein zweiter Sinn steht. Und daß es dieser unsichtbare Sinn ist, der jene Wahrheit sichtbar werden läßt, nach der wir uns alle sehnen" (7).

„Die unfreiwillige Gemeinschaft" (256) entstand durch einen das Ganze konstituierenden verdoppelten Zufall: in zwei nacheinander folgenden Luftangriffen wurde das Haus am Neuen Markt durch Bomben getroffen bis die im Keller Versammelten ,endgültig”, versehüttet wurden. Diese Verdoppelung des Zufalls, die Unwahrscheinlichkeit des Vorkommnisses, suggerieren von Anfang an einen Sinn, der sich hinter der ,bloßen” Zufälligkeit verbirgt. Es ist zwar im Text sehr oft von „Zufälligkeiten” (290), vom „,ächerlichen Zufall” (166), vom ,reinen Zufall” (242, 290, 301), von der ,Macht des Zufalls”" $(110,333)$ die Rede, doch die Behauptungen werden entweder sofort mit einem Fragezeichen versehen (333) oder das Auftreten des Zufalls ,symbolisch" (132) interpretiert. Die nicht verèinzelten Gedankengänge verschiedener Figuren der Handlung und des Erzählers (!) reichen von der Vermutung eines zweiten Bodens bis an die Überzeugung. Der Erzähler, der schon anfangs im Zufall die äußere Erscheinungsform der allgemeinen Gesetzmäßigkeit der Welt sah, überlegt, ob es wirklich nur ein lächerlicher Zufall war, der jene sieben Menschen zusammenkommen und die Bombe „,blind und dumm neben ihr Ziel” (166) fallen ließ: „Vielleicht war all dies gar nicht so lächerlich, vielleicht gab es den Zustand, den wir Zufall nennen, nicht. Vielleicht fiel jedem Ereignis, das uns widerfuhr, eine gewisse Wertung zu, ein gewisser Sinn?" (166).

Das, was der Erzähler erst begreifen mußte, um über Vermutungen zur Überzeugung zu gelangen, war für andere Personen der Handlung von vornherein klar. Schröder, der seine Weltkonzeption in Anlehnung an die Lehre Origenes konstruiert, hat mit ihr solche „Durchschlagskraft”, daß nicht nur er von einem hinter allen Dingen stehenden Sinn (236) spricht, sondern auch den Erzähler seine Theorie kommentarlos wiederholen läßt: „Das ganze Leben besteht aus Zufälligkeiten, die ein Mann namens Origenes im zweiten Jahrhundert nach Christus nach drei Gesichtspunkten hin zu untersuchen empfahl: nach einem historischen, einem symbolischen und einem metaphysischen" (290f).

Walter Faber (,,Es gibt keinen Zufall (...) das weiß ich jetzt. Das, was wir Zufall nennen, ist das Gesetz unseres Lebens, dem keiner entkommen kann”, 301) und Reinhold Gontard (,Der Zufall ist Schicksal. Er ist das Gesetz unseres Lebens. Nichts, was geschieht, ist sinnlos, alles ist vorgezeichnet", 348) präsentieren eine fast identische Meinung, die nun auch 
vom Erzähler endgültig angenommen und akzeptiert wird, indem er den $\mathrm{Zu}$ -Fall der Bombe kommentiert: „Sie fiel, wie sie fallen mußte” (241).

Einen Sinn hinter gehäuften Zufälligkeiten zu finden, bedeutet, eine Nachricht finden $\mathrm{zu}$ wollen, die nie gesendet worden ist. ${ }^{9}$ Wenn man es aber trotzdem versucht, muß diese Sinnfindung einen Sinn haben. Thomas Mann ließ Zéitblom den Zufall ins Schicksal uminterpretieren, um das falsche Bewußtsein bestimmter gesellschaftlicher Schichten in Deutschland zu entlarven ${ }^{10}$. Max Frisch tat es, um seinen Helden in Homo faber zurechtzuweisen und ihn für seine Schuld bestrafen zu lassen ${ }^{11}$. Worauf könnte die totale Fatalisierung in Simmels Roman zielen?

Die immer wiederkehrende Betonung des unzufälligen Zufalls läßt das Ganze in einer allegorischen Dimension erscheinen. "Alles, was hier geschieht" - sagte einmal Schröder - ,geschieht nur im Gleichnis"' (236). Dieses Gleichnis gipfelt in Schröders Tod: Als dieser gegen den Willen aller Beteiligten versucht, den Eingang des Kellers mit Sprengstoff freizulegen, wird er von Faber erschossen. Schröder, die Verkörperung des Faschismus, wird von einer strafenden Hand erreicht. Jeder von den Eingeschlossenen wußte zwar, daß der Chemiker gefährlich war, , aber keiner glaubte, daß er (ihnen) wirklich überlegen sein könnte" (279). Nahezu paradigmatisch sind die Reaktionen der Einzelnen auf Schröders Verhaltensweise. ,Wer von uns hat ernstlich etwas getan" - wird Susanne ausrufen - ,um Schröders Plan zu verhindern? Wer hat sich darauf vorbereitet, ihn zu verhindern? Keiner!" (279). Susanne selbst hatte vor ihm Angst gehabt, Faber hat sie ausgelacht, der Priester hat ihn unterschätzt und Fräulein Reimann geschlafen.

In diesem Zusammenhang wäre Fabers Schuß als ein symbolischer Akt des bisher ausgebliebenen bewußten Widerstandes anzusehen. Wäre, denn auf die Frage, warum er den Nazi erschossen habe, antwortet Faber mit,,Ich weiß es nicht" (278); nach dem Verlauf der Dinge gefragt, weiß er nur mit „Dann weiß ich nichts mehr" (279) zu antworten. Somit wird Fabers Tat zu einer unbewußten Reflexhandlung und er selbst zum Werkzeug jenes schon erwähnten verborgenen Sinns, der die Welt des Romans vorbestimmt und regiert. Es fragt sich nur, warum das Schicksal auf einmal das Böse bestraft, nachdem es dieses Böse jahrelang toben ließ.

Auf diese Frage gibt der Text keine Antwort. Es kommt einfach so.

- Die auffallende Häufung der Zufälligkeiten verringert gleichzeitig den Grad ihrer Wahrscheinlichkeit und erweckt somit den Schein eines verborgenen Sinns, einer Gesetzmäßigkeit.

10 Vgl. dazu: W. Bialik, Der Zufall als historiosophische Kategorie. Thomas Mann: „Doktor Faustus", in: derselbe, Die Ästhetisierung der Kategorie des Zufalls im deutschsprachigen Roman des 20. Jahrhunderts; Poznań 1978, S. 57-66.

11 Vgl. W. Bialik, Der Zufall als Strafe. Max Frisch: ,Homo faber”, ebenda, S.8290. 
Gontard erklärt Susanne sein neues Glaubensbekenntnis und vergleicht es mit dem Schröders: „Sie müssen lernen, wieder zu glauben. Darin war Schröder uns voran. Er glaubte bedingungslos an eine schlechte Sache. Aber er glaubte an sie. Deshalb war er stark zu einer Zeit, in der wir noch zögerten, weil wir nicht wußten, wofür wir kämpfen sollten, weil wir zwar gegen eine Idee, aber nicht für eine andere lebten. (...) Ich glaube an die Gesetzmäßigkeit des Lebens, die viele Menschen Zufall nennen. An eine progressive Entwicklung der Welt, die in positive oder negative Bahnen zu lenken bei uns liegt. (...) Es handelt sich (...) schon lange nicht mehr um das, was wir glauben, hoffen oder fürchten. Es handelt sich allein, um das was wir tun. Was andere Menschen tun" (350). Also kämpfen oder glauben? Gontards Konversion, die wohl in einer direkten Relation zur symbolisch-schicksalshaft ausgelegten Tat Fabers steht, hat zur Folge, daß er sich in dem zwischen der Vorbestimmtheit und der aktiven Mitgestaltung des Weltgeschehens gespannten Netz hoffnungslos verstrickt. Er sagt zwar: „Was wir heute tun, trägt morgen Früchte, und es sind Früchte unserer Taten von gestern, die uns heute bedrücken" (349), aber unmittelbar davor heißt es: „Was dann, durch uns, geschah, kann man nicht Zufall nennen. Denn es konnte nichts anderes geschehen. Und es wird auch in Zukunft nichts anders geschehen können, als wie es eben geschieht" (349).

In diesem Kontext erscheint Gontards Aufruf zur menschlichen Aktivität fragwürdig. Die Aktivierung des Menschen zum Mitgestalter und Veränderer der Welt ${ }^{12}$ steht im krassen Widerspruch zur vorbestimmt ausgegebenen Welt. Gontards Konzeption ist bei ihrer ganzen Verzwicktheit nichts anderes als der Übergang vom Schicksalsglauben überhaupt zum - durch nichts begründeten - Glauben an das gute Schicksal. Der bedingungslose Glaube ermöglichte es Schröder, seine bösen Pläne zu realisieren, der starke Glaube soll auch die Pläne Gontards und der ihm Gleichgesinnten möglich machen. Entscheidend dabei ist nur die Intensivität des Glaubens, und das ganze Gerede über die schöpferische Kraft des Menschen erscheint als bloße Phrasendrescherei.

Einiges kann man Simmel jedoch nicht absprechen. Sein Schnitt durch die deutsche Gesellschaft des Jahres 1945 machte Denkweisen und Haltungen sichtbar, die für diese Gesellschaft kennzeichnend waren: ein Nazi, der blind an seine Botschaft glaubt, ein Priester, in dessen Weltanschauung es keinen Platz für Gott mehr gibt, eine alte Frau, die nur überleben will, zwei junge,

12 Brüggemann hat Recht, wenn er sagt, daß die Reichweite der Veränderung nie über das Individuum hinausreicht: „,Wenn Sie beide zusammenbleiben, ist schon viel getan. Sie würden richtig handeln" (295), erklärt Gontard Walter Faber und Susanne die „Aktivität” ihres weiteren Lebens. Wie dieses „Handeln” aussehen soll, sagt er nicht. Nicht zu vergessen ist es aber, daß das - wiederholen wir - die Perspektive der Roman. figuren ist. 
die sich nach Sicherheit und Liebe sehnen, ein Soldat, der zur Schlußfolgerung kommt, daß man auch mit dem Schweigen morden kann und ein Kind, das schon erwachsen ist, weil es schon gut lügen kann (298). Daß alle diese Menschen einschließlich Schröder - im Grunde genommen hilflos der Welt ausgeliefert waren und bis zum Schluß keinen Ausweg wußten, ist nicht die Schwäche des Romans - wie es Brüggemann sieht - sondern die Schwäche der damaligen deutschen Gesellschaft, die doch von außen her befreit werden mußte. Simmels Verdienst ist es, diese Schwäche bloßzulegen: es ist nicht sein unbeholfener „,moralischer Antifaschismus”, der hier zum Vorschein kommt, sondern der ,seiner" Figuren ${ }^{13}$.

Stellte Simmels erster Roman vor allem einen Versuch dar, eine Art Abrechnung mit der jüngsten Vergangenheit vorzunehmen, so verlegt sich der Schwerpunkt des zweiten Romans auf die möglichen weiteren Entwicklungsperspektiven des Landes und der Gesellschaft.

Das geheime Brot (1950) führt den Leser in eine typische Trümmer-Landschaft der ersten Nachkriegszeit ein. Die im Ton einer grau-grauenvollen Berichterstattung gehaltene Exposition beginnt mit der Beschreibung Wiens des Jahres 1946 oder 1947, gipfelt im breitest möglichen Rahmen, einer Art Weltspiegel, dessen Abbildungsfläche sich dann allmählich wieder verringert und die Welt auf die Stadt Wien und weite Textstrecken lang gar auf ein verfallenes Haus in Wien reduziert. Das letzte Glied der Wien-Welt-Wien-Reihe unterscheidet sich grundsätzlich von dem ersten: die anfängliche anonyme Masse der Unbehausten, Hungernden und Verzweifelten ,gebiert" Individuen, auch unbehaust, hungernd und verzweifelt, doch gewissermaßen „privat" und - obwohl auch typisch - einmalig.

In dieser von der Untergangsstimmung erfüllten Welt des Provisorischen, wo es ,,keine Echtheit der Empfindung mehr, keine Tiefe des Gefünls, nur noch Oberflächlichkeit, Verzweiflung, Langeweile und Ekel" (11) gab, taucht Jakob Steiner auf, „eine umgefallene Vogelscheuche” (12), die seine Lebensbilanz zieht: ,,verloren: Frau, Kind, Wohnung, Arbeit, Hoffnung; gewonnen: Die Vermutung, daß die Welt sehr häßlich geworden ist; Zukunftsaussichten: Einsamkeit, Hunger, Kälte, Krieg”' (13f).

Auch die Aufstellung der Aktiva und Passiva der beiden anderen Helden des Romans weicht nur im Konkreten von der Steiners ab: die alte Frau Magdalena Huber hat ihren Mann verloren, ihr einziger Sohn ist während des. Krieges verschollen; Herr Aram Mamoulian, die führende Persönlichkeit

13 Wenn man unter Umständen dem Autor Simmel den Vorwurf machen kann, er selbst vertritt die Konzeption der Vorbestimmtheit des Weltgeschehens, da er seinen auktorialen Erzähler diese Konzeption mehrmals ,unterstützen" läßt (vgl. die Anm. 4), so ist es völlig verfehlt, ihn mit seinen Figuren zu identifizieren, da kein einziger Grund vorliegt, eine von ihnen als porte parole des Autors zu betrachten. 
des Werkes (hat Jakob von dessen Selbstmordabsichten abgebracht) vergleicht seine Lage mit der des Lebensmüden: „hören Sie zu: Sie haben ihre Wohnung verloren. Nun, das habe ich auch. Sie haben ihren Beruf verloren. Nun, das habe ich auch. Ihr Kind ist gestorben. Ich habe niemals Kinder gehabt. Sie besitzen kein Geld. Ich besitze auch keines. Ihre Frau ist gestorben. Ich habe niemals eine Frau gehabt. Und jener Mensch, den ich liebte, ist nicht gestorben. Aber ich bin für ihn gestorben (...) und das ist auch nicht das angenehmste" (48).

Mit dem Erscheinen Mamoulians, der immer nach Glück suchte, und dem immer nur Vergnügen begegnet war (er hatte eine luxuriöse Villa, ein großes Vermögen und viele Freunde, die dann "seltsamerweise" - nachdem der Krieg ihm alles Materielle genommen hatte - verschwanden), beginnt das schöne Märchen von einer besseren Zeit, die man sich selbst erarbeiten muß. Wunderbarerweise $^{14}$ ließ sich Herr Mamoulian dreierlei niemals nehmen. Der auktoriale Erzähler bezeichnet das als „Mut, Hoffnung und Humor”(43): Er fragt nicht nur ,nach dem Sinn dieses seltsamen Karnevals” (10), sondern findet ihn, er gehört zu jenen, ,die über ihrem Hunger nicht ihren Verstand verloren haben" und beschließt - in das Symbolhafte steigend - „von dem geheimen Brot (zu) leben, das aus den Furchen der Armen wächst" (47), wobei dieses ,geheime Brot" mehr als Triebkraft des konkreten Tuns zu verstehen ist und nicht - wie z. B. bei Böll ${ }^{15}$ - eine magische Größe, die die zwischenmenschlichen „naturellen" Beziehungen der ersten Nachkriegsjahre kennzeichnete.

Mamoulians ziemlich abstrakte Hoffnung läßt ihn nicht passiv sein: er übersetzt ein Buch seines Freundes, er arbeitet. Im Gespräch mit Steiner, der die Revolutionen haßt, weil sie verdummend wirken und üble Instinkte fördern (82), plädiert er für die revolutionäre Entwicklung: ,Ich liebe Revolutionen (...) und ich hoffe sehr, daß jene große und endgültige Revolution, der wir entgegentreiben, sich noch zu meinen Lebzeiten abspielen wird. (...) Ich will ein buntes Tuch um meinen Hals winden und andere Menschen an den Händen halten und ,Bruder' zu ihnen sagen und mit ihnen um die Freiheit und das Recht kämpfen. (...) Um die Freiheit der Rede und des Glaubens (...) und um die Freiheit von Hunger und Furcht" (82). Umsomehr verwundert eine spätere Meinung Mamoulians, in der er die obenzitierte als ,,ironisch

14 Die Atmosphäre des Wunderbaren, Außergewöhnlichen und Geheimnisvollen durchzieht den ganzen Roman. Es wird vom ,geheimnisvollen und tiefen Sinn" (43) gesprochen, der hinter den Ereignissen steht, von einer "sehr geheimnisvollen Weise" (249), vom „Wunder" (117, 272), „Verhängnis" (191), ,sonderbar" (305, 306) und „unglaublich" (269). Es wird endlich expressis verbis behauptet, daß der Roman kein „,normaler Roman” (120) ist: „Es wird (...) dem geschätzten Leser schon aufgefallen sein, daß dieser Roman weit davon entfernt ist, normal zu sein..." (120).

15 Vgl. H. Böll, Das Brot der früher Jahre, Köln 1955. 
gefärbte” (121) aufzuheben versucht: „Eine Revolution ist kein aufbauender Vorgang. Eine Revolution verpflichtet zu nichts. Eine Revolution hat noch nie ein anständiges Haus aus einer Ruine gemacht. Immer bloß umgekehrt. Mit einer Revolution schaffen die Menschen sich nur ein bißchen Luft, um so wie vorher weiterleben zu können" (121). Die obige Widersprüchlichkeit ist auf Mamoulians innere Zerrissenheit zurückzuführen: einerseits will er um jeden Preis aktiv sein, andererseits assoziert er jede Aktivität mit immer mehr anwachsenden Verpflichtungen, von denen er - die offensichtliche Lehre des sog. Lebens - nicht hören will: er hat gelernt, daß das friedliche und wohlwollende Nebeneinanderleben nicht so gefährlich und vor allem ergiebiger als das Miteinander ist. „Die Ordnung in das Chaos zu bringen” (121), ist nichts für ihn, zumal es sich einfach nicht lohnt, die Welt wieder zu ordnen, denn ,wie lange kann es schon dauern, bis ein neuer Krieg kommt?" (122). Daß der auf den ersten Blick so konsequente und souverän wirkende Armenier ununterbrochen zwischen extremen Auslegungsmöglichkeiten lebenswichtigster Probleme schwingt, daß er die ganze Zeit erst ge- oder umgeformt wird, beweist gerade seine Einstellung zum Krieg. „Es wird keinen Krieg geben” - sagt er einige Zeit später. „Ich habe es mir überlegt. (...) es kann keinen Krieg geben, weil der Krieg tot ist. (...) Das Zeitalter des Soldatentums hat bei Hiroshima sein Ende gefunden. Die Wissenschaftler haben die Generale besiegt. (...) Ich muß mich, um deulicher zu werden, eines Paradoxons bedienen: der kommende Krieg, an den ich nicht mehr glaube, wird so furchtbar sein, daß er nicht kommen wird. Er wird nicht kommen, weil er so furchtbar sein wird. Er wird nicht kommen, weil er der absolute Schlußpunkt in der Geschichte unserer Welt wäre" (246).

Die Tatsache, daß die Menschheit über ein so enormes Vernichtungspotential verfügt, veranlaßt Aram Mamoulian - ganz anders als bei Dürrenmatt ${ }^{16}$ - den Glauben an die Vernunft des Menschen zu manifestieren und davon den Sinn des menschlichen Tuns abzuleiten: „Es wird keinen neuen Krieg geben, nicht, weil die Mächtigen dieser Erde nicht infam genug, nicht, weil die Generale nicht borniert genug, nicht, weil die Rüstungsfabrikanten nicht gerissen genug, sondern weil sie alle nicht dumm genug sind, um es zu ihm kommen zu lassen" (246).

Dieser schöne Glaube an die menschliche Vernunft, die von seinem Widersacher, Steinert, nicht geteilt wird (,Militärs haben von vornherein keinen Verstand, und Politiker, sobald sie an die Macht kommen, verlieren das letzte bißchen", 246), bleibt eben nur ein Glaube. Daß dieser Glaube

16 Von derselben Prämisse ausgehend, bekunden Dürrenmatts Helden (oder Erzähler) gerade sein Mißvertrauen, und das nicht nur gegenüber der menschlichen Vernunft (Physiker), sondern auch in Hinsicht auf die Welt, deren Beschaffenheit (Zufallsbedingtheit) sie entweder einem grenzenlosen Nihilismus (Emmenberger und Marlock im Verdacht) oder Fatalismus (der Er zähler des ersten Teiles der Panne) verfallen läßt. 
eigentlich einer Wunschvorstellung entspringt, bezeugt die spontane Äußerung Mamoulians gegen Schluß der Diskussion: „,Wir brauchen keine Überwachung der Bombe! Wir brauchen eine Überwachung des Menschen!" (249).

Den Anlaß der ersten der zahlreichen Diskussionen des Romans bildete der unerwartete Vorschlag Steiners, Mamoulians Ruine wiederaufzubauen, in der alle drei (Mamoulian, Steiner und Frau Huber) seit ihrem Treffen wohnten. Vorher jedoch, am Ostersonntag, wurde Herr Mamoulian 'von Josephine Werner und ihrer Tochter Ruth besucht. Da der Gastgeber „unbedingt jemanden brauchte, für den er ,da' sein, um den er sich Sorgen und dem er Geschenke machen konnte" (89), ist es nicht verwunderlich, daß er die kleine Ruth, deren Mutter nach dem Tod ihres Mannes gezwungen war, auf die Straße zu gehen, in sein Herz schloß. ,Jakob Steiner war bezaubert von dem Liebreiz und der Schönheit Josephine Werners" (85), in ihr erblickte er plötzlich die Chance, wieder das zu gewinnen, was er verloren hat: Frau, Kind, Glück und Frieden. Auf diese Chance ist Steiners Vorschlag, die Ruine wiederaufzubauen, zurückzuführen. Nach einigem Hin und Her (Mamoulian betrachtete den Wiederaufbau als Versuch, Ordnung in das Chaos zu bringen, und war zuerst - wenigstens scheinbar ${ }^{17}$ - dagegen) war die Sache beschlossen: Um Geld für den Bau zu beschaffen, sollten alle drei halbtags arbeiten: Frau Huber, gelernter Maurer, auf den Bauplätzen des Herrn Lobgesang, Mamoulian, indem er weiter für Zeitungen schrieb, und Steiner als Schokoladen-, Hosenträger- und Zigarettenverkäufer. In der übrigen Zeit sollten sie zusammen die Ruine wiederaufbauen: Frau Huber war doch Maurer, Steinert Tischler von Beruf, und Mamoulian hatte die Ruine.

Wie Mamoulian von vornherein vermutete, wurden die sich bisher selbst Überlassenen zu einem interessanten Fall für die zuständigen Behörden. „,... jetzt sind Sie” - erklärt der Armenier Frau Màgdalena - „eine gewichtige Persönlichkeit mit einem kapitalistischen Air, wenn Sie auch keinen Groschen Geld besitzen. (...) Aber Sie sind ein selbständiger Mensch geworden, Sie verfolgen Ihre eigenen Pläne, Sie wollen etwas, Sie bezwecken etwas, und damit sind Sie eine unbedingt zu kontrollierende Persönlichkeit geworden, für die sich die Behörden auf einmal brennend interessieren. (...) Sie sind unter schöpferische Menschen gegangen, (...) unter jene, die es sich in den Kopf gesetzt haben, einen Zustand zu ändern. Sie haben etwas angeregt, das Kreise ziehen kann - und plötzlich werden Sie von der Behörde mit mißtrauischem Interesse betrachtet, denn der Behörde sind schöpferische Menschen immer unheimlich" (131f). In diesem Kontext ist Mamoulians Verwunderung über die Vorladung zur Baupolizei mehr gespielt als echt: er erfährt ,über-

17 Mamoulians Uninteressiertheit ist gespielt und Ausdruck seiner später formulierten Vorsichtigkeit gegenüber allem, was ,,revolutionär" ist. In "Wirklichkeit" hat auch bei ihm der Vorschlag sofort Anklang gefunden: „Also, wie habt ihr euch denn die ganze Geschichte vorgestellt? Erzählt doch einmal (...) ich bin sehr neugierig" (125). 
rascht", daß eine Anzeige gegen ihn wegen des ,wilden" Baus eingegangen ist: "Sie setzen sich einfach in den Kopf, Ihr Haus wieder aufzubauen" klagt der Beamte an - ,und beschäftigen dabei zwei völlig ungeeignẹte Arbeitskräfte und bauen, wie mir scheint, selbst mit..." (184). Als Mamoulian nicht umhin konnte zu sagen, ,er beneide die Beamten der Baupolizei um ihre Sorgen” (184), parierte der Polizist: „Und wenn wir uns nicht um Sie kümmerten? Was würde geschehen? Sie würden weiterbauen, und eines Tages würde Ihnen das Dach auf den Kopf fallen!" (185). Die Tatsache, daß sich die Baupolizei für Mamoulian nicht interessierte, als ihm das Dach zum ersten Mal auf den Kopf fiel, daß es für sie auch uninteressant blieb, ob sich die Drei ,jetzt", zu der Zeit, in der sie die Ruine bewohnten, nicht in Lebensgefahr befanden, wird von dem Beamten nicht zur Kenntnis genommen: es gehört nicht in die gerade angelegte Akte.

Von den Behörden gezwungen (Der Beamte: ,Außerdem ist Ihnen im Falle einer Weiterführung Ihres Planes selbstverständlich jede Unterstützung von seiten der Behörden verschlossen" (187), versucht Mamoulian, das erforderliche Material zu „organisieren”: Frau Huber sichert sich die Hilfe ihres Arbeitgebers, des schon erwähnten, in den ersten Nachkriegsjahren reich gewordenen ehemaligen Schlächters und jetzigen Bauunternehmers Lobgesangs, der - in seinem neuen Neureichen-Milieu unglücklich - Vieles verspricht unter den einzigen Bedingung, ihn von Zeit zu Zeit mitbauen und im Keller zusammen mit den anderen schlafen zu lassen. Auch die Männer sehen nicht zu und stehlen 12 Säcke Zement, der sich später als für Schwarzmarkt-Schiebungen getarnter Zucker erweist. Obwohl sie sich selbst anzeigen, in der Hoffnung, daß die Polizei die ,anständigen Motive bei diesem Diebstahl würdigen" (212) wird, landet Mamoulian hinter Gittern. Erst eine gut organisierte Solidaritätsaktion („,disziplinierte Demonstration”, 224) der einbeinigen Straßenverkäufer (Steiners Arbeitskollegen) hilft ihm wieder, auf freien $\mathrm{Fuß}$ zu kommen.

Die Halbtagsarbeit Steiners sowie die Schwierigkeiten mit der Beschaffung des Baumaterials ermöglichen Simmel, seinen Leser in das aktuellste Zeitgeschehen (der Roman ist 1950 erschienen) einzuführen: Schwarzmarkthandel, Lebenswege und Aufstiegsmöglichkeiten der Vorläufer des Wirtschaftswunders ${ }^{18}$, Wiederaufleben der Bürokratie, Ratlosigkeit des kleinen

18 Der erste Eindruck des Lesers, er befände sich in einer hoffnungslosen Welt, der Armen und Verzweifelten, wird mit unscheinbaren, gewissermaßen en passant eingeflochtenen Textpassagen konfrontiert, die bezeugen, daß es schon in den ersten Nachkriegsjahren zwei Welten in Deutschland gab. „Jakob Steiner" - heißt es am Anfang: des Romans - „machte einen verkommenen Eindruck auf die feinen Leute, die in den schönen Villen der Gegend wohnten...” (12). An einer anderen Stelle ist zu lesen: „Herr Mamoulian sieht sehr alt und verloren aus zwischen den großen und vornehmen Automobilen und den fröhlichen, eiligen Menschen..." (69). Tobias Lobgesang, einer der 
Mannes gegenüber den allmächtigen Behörden, Heimkehrertransporte, all das placiert Das geheime Brot mitten in der Aktualität: der Zeitbericht wird oft zur Zeitkritik.

Diese Zeitkritik überschreitet bei Simmel jedoch nie die Grenze der Berichterstattung: dort, wo sie sich dem Postulativen eröffnet, 'verliert sie den Boden der Wirklichkeit und mündet im Märchenhaft-Utopischen. Der Bau des Hauses zum Symbol des zu erbauenden Neuen geworden, zum Symbol des neuen Menschen und der neuen Wirklichkeit, bedarf leider eines W unders, um vollendet zu werden: als eine Art Deus ex machina taucht plötzlich Mamoulians Landsmann Reuben Tschipourian auf, der ihm - zugleich Auftraggeber und Freund - eine beträchtliche Summe für die Übersetzung seines Buches überreicht. Die von Tschipourian vorgeschlagene Mitarbeit (Arams Freund ist ein superreicher Geschäftsmann, ein Prototyp des Simmelschen Weltreisenden) auf später verschiebend, wendet sich Mamoulian der Vollendung seiner Idee zu: zuerst muß das Haus, das ,etwas wie eine Bank der guten Hoffnung, der anständigen Instinkte, der Ehrlichkeit und des guten Willens" (279) ist, stehen. In diesem von der Mustergesellschaft bewohnten Musterhaus „,soll es keine Geheimnisse geben" (281), hier steckt eine idyllische Welt im Keim, in der nichts von nichts getrübt wird, wo Gerechtigkeit waltet und Klassenunterschiede verschwinden: da ,der erste Mensch, der daran dachte, irgendeinen sinnvollen Griff auszuführen, eine Synthese von Faustund Stirnmensch, sozusagen der vollkommene Mensch war, der erst später in zwei Komponenten zerfiel" (171), da also die geistige Arbeit sich gegenüber der körperlichen so verhält wie das Ei zur Henne (169-173), leben im neugebauten Haus in einem einträchtigen Miteinander Arbeiter und Intellektueller ${ }^{19}$ : der strahlende Anfang der später so hochgepriesenen - und nicht verwirklichten - Partnergesellsch aft, die im Roman insofern über das Haus hinaus reicht, als in ihm alle, die beim Bau beholfen haben, herzlich willkommen sind..$^{20}$

Nutznießer des Nachkriegschaos ist erster der ganzen Reihe der Simmelschen Wirtschaftswundertäter: Durch geschickte Operationen wurde er schnell reich und... unglücklich. Aus seinem Milieu herausgerissen, von seiner Parvenüfrau terrorisiert, sehnt er sich nach Arbeit und nicht mehr nach Geldmachen (vgl. S. 134-150). Die Unterscheidung zwischen der ,eigentlichen" Arbeit und Geldmachen kommt noch krasser bei Mamoulian zum Ausdruck, der nur und allein den Bau des Hauses als „Arbeit" ansieht, alles andere, auch das, was Geld für den Bau einbringen soll (seine Zeitungsartikel, Halbtagsarbeiten der Frau Magdalena und Steiners), als "Geldverdienen” (128).

10 Viel zu denken gibt hier die Tatsache, daß Herr Mamoulian - der Intelektuelle bis zum Ende des Romans Jakob Steiner - dèn Arbeiter - siezt, und das ungeachtet dessen, daß hier ununterbrochen von Familie, Gemeinschaft und Partnerschaft die Rede ist.

${ }^{20} \mathrm{Da}$ in dieser heilen Welt jedes Verdienst, jede gute Tat belohnt wird, vergißt Herr Mamoulian natürlich nicht, seine ehemälige Gèliebte; die Prostituierte Yvonne, 
Noch ist der Wunder nicht genug: der überdimensionale Mamoulian verfügt über die wundersame Gabe, den kommenden Tod der. Menschen vorauszufühlen; durch den dreifachen Traum der Mutter angesagt, kehrt aus Rußland der verschollene Sohn der Frau Magdalena zurück; als Symbol des neuen Lebens, der glücklichen Zukunft der entflammten Liebe zwischen Josephine und Steinert, erblüht mitten im Winter der hoffnungslos verdorrte Rosenstrauch vor dem Haus.

Simmels Verfahrensweise entspringt einer sehr intensiven Wunschvorstellung, die wiederum einen Traum entștehen läßt. Da dieser Traum in den Voraussetzungen Deutschlands der ersten Nachkriegsjahre keine Aussichten hat, Wirklichkeit zu werden, bleibt nur zu hoffen, das er mal doch verwirklicht wird. Im Gla uben an die bessere Zukunft - charakteristisch sowohl für christliche Schriftsteller wie auch für Anna Seghers ${ }^{21}$ - und in der mit ihr verbundenen Hoffnung, liegt - nach Simmel - die einzige Macht; die Wunder wirkt, Wunder, die einzig und allein imstande sind, die trübe Wirklichkeit in ein Märchenland zu verwandeln. Eine andere Möglichkeit für die Generation der damals Erwachsenen sieht der Autor nicht: er läßt seinen Träumer Mamoulian, der gelernt hat, , daß es nur eine Sünde im Leben gibt, und das ist: die Hoffnung verlieren" (306), glauben, hoffen und lieben, was notwendigerweise zur Passivität und Anerkennung des Bestehenden provoziert: "Nur die Liebe ist sinnvoll" - sagt Mamoulian - ,jeder Haß ist sinnlos. Alle Wesen des Kosmos sind unsere Brüder, wir brauchen die Welt, so wie die Welt braucht uns, wir müssen uns mit ihr versöhnen wie mit dem Meer und dem Wind, dem Älterwerden, dem Glück und der Einsamkeit (...) mit dem Guten und dem Bösen - mit Engeln und Haifischen ${ }^{22}$. Wollten wir sie

einzuladen (313), die am Anfang des Romans als einzige sofort bereit war, ihm Geld für das Ostergeschenk für Ruth zu borgen.

21 Anna Seghers, die in Die Toten bleiben jung einem noch ungeborenen Kind die Fortsetzung der revolutionären Tätigkeit seines Vaters ,anvertraute", brachte auch nur und allein ihren Glauben an das neue Deutschland zum Ausdruck. Thre fatalistisch-biologistische Vision ist darauf zurückzuführen, daß auch sie in der Zeit, in der der Roman entstand, noch nicht wußte, wie die zukünftige gesellschaftlich-politische Lösung zu erreichen ist. So brachte sie wenigstens ihre Überzeugung und Wunsch zum Ausdruck. Vgl. dazu W. Bialik, Profundes Gesellschaftsbild und problematische Historiosophie. Anna Seghers: „Das siebte Kreuz" und „Die Toten bleiben jung”, in: derselbe, Die Ästhetisierung der Kategorie des Zufalls im deutschsprachigen Roman des 20. Jahrhunderts, Poznań 1978, S. 67-76.

22 Dies ist eine der problematischsten Stellen des Romans. Indem Mamoulian Meer, Wind und Älterwerden einerseits, Glück und Einsamkeit andererseits gleichsetzt, als Welt ausgibt und zur Versöhnung mit dieser Welt aufruft, identifiziert er Naturereignisse, auf die der Mensch keinen oder nur begrenzten Einfluß hat, mit sozialen (psychologischen) Fakten, die nur und allein von dem Menschen selbst abhängen oder abhängen sollen. Dieso. Identifizierung hat zur Folge, daß in seinen Augen auch menschliches Glück (oder Unglück) und Einsamkeit (oder Nicht-Einsamkeit); natur-oder gottgewollt und somit 
hassen, so haßten wir Gott, in dessen Schöpfung sie alle ihren Platz einnehmen. Wollten wir sie hassen, dann wäre ein bestimmter $\mathrm{Ha}$ auf ein bestimmtes Objekt gänzlich sinnlos, dann müßten wir die Welt als solche hassen, den Engel ebensogut wie den Haifisch, denn beide wurden von derselben Hand geschaffen." (307).

In der Welt, wo man vom ,geheimen Brot" zehrt, wird jeder echte Glaube, jede tiefe Hoffnung über kurz oder lang mit dem Wunder belohnt: Aram glaubte an seinen Freund Tschipourian, Frau Huber an die Rückkehr ihres Sohnes, Steiner daran, daß die verlorene Liebe (der Rosenstrauch) wieder einmal erblüht.

Die Aktivität des Menschen fördernden Gedanken Mamoulians dürfen nicht irreführen: das Wunder, auf das zu warten der Mensch verurteilt ist, liegt außerhalb der menschlichen Einflußsphäre. Der Armenier sagt doch: „Der Mensch ist gut. (...) ich finde, daß auf eine sehr geheimnisvolle Weise immer öfter und immer deutlicher das Gute über das Böse siegt, und ich bin fest entschlossen, daran zu glauben, daß es allein von uns abhängt, ob wir in Frieden leben und glücklich sein werden" (249). Die scheinbare menschliche Möglichkeit, die Welt zu formen, steht hier im krassen Widerspruch zur "geheimnisvollen Weise", auf die das geschehen soll. Und hier treten wir auf den unsicheren Boden der Weltanschauung des Romans: Sosehr sich Simmels Held bemüht, die Aktivität des Menschen hochzupreisen, es ist nicht die Macht des Menschen, die die Welt des Romans regiert.

Schon am Anfang des zweiten Kapitels wird Kants Formulierung „List der Vernunft" zitiert, was auf die Konzeption der - in welchem Grade auch immer - zufallsbedingten Entwicklung der Welt schließen läßt, um gleich im nächsten Satz, wo vom "schicksalsmäßigen Ablauf gewisser Ereignisse" (30) die Rede ist, eine übernatürliche, dem ,säkularisierten" Zufall entgegengesetzte Prägung zu erhalten. Auch im weiteren Verlauf des Textes werden Begebenheiten als ,mehr als Zufall" (43) ausgelegt und es wird auf eine überirdische Instanz hingewiesen, die mal als Leben, mal als Verhängnis oder Vorsehung, mal als der liebe Gott erscheint. Neben der an Döblins Berlin Alexanderplat_ erinnernden Vorausdeutung: ,Und nun stand das Verhängnis schon riesengroß wie ein maßlos aufgerichteter Schatten hinter ihm und hob sich in den hohen Himmel hinein wie ein böser Geist und war bereit zuzuschlagen" $(191)^{23}$, die nur die die menschliche Freiheit in Frage stellende

unanfechtbar sind. Dieser Aufruf bedeutet also nicht anderes als Würdigung der Passivität und Betrachtung der Wirklichkeit als ein für alle Mal gegeben und unveränderbar.

23 Wie Döblin in Berlin Alexanderplatz, stellt Simmel jedem Kapitel eine stichwortartige kommentierende Inhaltsangabe voran. Die hier zitierte Stelle, die sowohl im 'Stilistisch-Wörtlichen als auch im Inhaltlichen mit den, ,schicksalhaften" Bemerkungen Döblins in bezug auf Franz Biberkops Ǎhnlichkeiten aufweist, fungiert - obwohl ein abschließender und nicht einleitender Kommentar - genau wie Döblins Vor. 
Existenz einer höheren Macht andeutet, taucht im Text auch eine Art Warnung vor dem Überschreiten der irdischen Kompetenzen auf: „Es ist eine alte Tatsache, daß immer dann etwas Böses geschieht, wenn zu lange Gutes geschehen ist. Auch auf diese Weise reguliert sich der Lauf der Welt. Herr Mamoulian kannte diese regelmäßigste aller Sinuskurven ganz genau. Er wußte, daß es sich nicht lohnte, allzu traurig über ein. Unglück zu sein, weil das Glück schon auf dem Fuße folgte. Und er wußte auch, daß es töricht war, sich über dieses folgende Glück allzusehr zu freuen(...) Obwohl Herr Mamoulian aber, in Kenntnis dieser verwickelten Tatsachen, sich sein Leben lang darum bemühte, in einer Mittellage, in einem Schwebezustand zwischen diesen beiden Extremen, zu verharren, gelang es $\mathrm{ihm}$ doch niemals (...) Das Leben ließ Herrn Mamoulian für seine Erkenntnisse zahlen, weil es richtige Erkenntnisse waren - so wie das Leben alle zahlen läßt,. die im Begriff stehen, ihm auf die Schliche zu kommen" (179f).

Die ,säkularisierte Christlichkeit" 24 ist in diesem Kontext nur scheinbar oder nur in einem begrenzten Rahmen säkularisiert: Auch der im Roman als Statthalter Gottes auf Erden fungierende Mamoulian (oder vielleicht gerade er) wird zur Rechenschaft gezogen, wenn er bestimmte „Weisungen” und „Richtlinien” nicht beachtet. Die Abhängigkeit von der vielgesichtigen Macht ist hier nicht total, sie läßt dem Menschen einen gewissen Spielraum. Nun kommt es darauf an, diesen Spielraum nähèr zu bestimmen.

Zuvor war von der widerspruchsvollen Einstellung Mamoulians zur revolutionären Entwicklung die Rede: „Einmal (...) behauptete er, daß er sich nach einer großen Revolution sehne, daß er Fahnen tragen und auf die Barrikaden klettern wolle, und gestern erzählte er mir, daß uns nur mit Weisheit, Ruhe und großer Geduld zu helfen ist"' (309). Die scheinbare Polarität der Meinungen Mamoulians kann dadurch aufgehoben werden, daß sie zeitlich verschieden legen, daß sie eine Zeit trennt. Der Armenier hat eine Entwicklung(?) durchgemacht von der spontanen revolutionären Haltung, deren er sich übrigens später schämt und als ironisiert abzuschaffen versucht, zur Überzeugung über die Richtigkeit der von Gott zugelassenen evolutionsmäßigen Entwicklung in Richtung auf die bessere Welt: er wird allmählich gegen alle oberflächlichen Formulierungen - zum Garanten der Ruhe, griffe und sorgt für einen flüssigen Übergang zur, - wieder ,döblinischen" - Úberschrift des nächsten Kapitels: „Das achte Kapitel, in welchem die Herren Mamoulian und Steiner blindlings in ihr Verhängnis rennen" (192). Und bei Döblin: „Siebentes Buch: Hier saust der Hammer, der Hammer gegen Franz Biberkopf” oder „Achtes Buch: Es hat nichts genutzt. Es hat noch immer nichts genutzt. Franz Biberkopf hat den Schlag erhalten, er weiß, daß er verloren ist, er weiß noch immer nicht, warum". (A. Döblin, Berlin Alexanderplatz. Die Geschichte vom Franz Biberkopf, Berlin 1930, S. 347 u. 411 ).

s4 A. Weber, Das Phänomen Simmel. Zur Rezeption eines Bestseller-Autors unter Schülern und im Literaturunterricht, Freiburg i. B. 1977, S. 117. 
zum - wie Tschipourian ihn nennt - „Friede Gottes” (310): er rät Steiner, geduldig, schrittweise und langsam seine Ziele zu verfolgen und , keine Sprünge in die Zukunft zu machen" (268). Daß er zugleich porte parole Simmels ist, beweist eine Art gemäße Haltung einer anderen durchaus positiven Figur der Handlung, des einbeinigen Schwarzmarkthändlers Olbrich. Die von ihm organisierte, einzige Demonstration des Romans, eine Aufruhr gegen die Ungerechtigkeit, ist - jetzt verständlicherweise - ,eine kleine disziplinierte Demonstration" (224), die nicht schaden kann. Und sie ist erfolgreich.

Es wäre jedoch ungerecht, hier stehenzubleiben und Simmels Weltkonzeption mit dem allmächtigen Gott zu identifizieren. Im Gegenteil: das Herbeiziehen Gottes wurde - mag das paradox klingen - einerseits von der Wirklichkeit, andererseits von der Vision der neuen Wirklichkeit erzwungen. Gott waltet über die Simmelsche Welt zeitweilig, als Ersatz-Mensch. Der Schriftsteller „,bedient sich” Gottes, weil die Menschen noch nicht reif genug sind, selbst „Wunder" zu vollziehen und Märchenländer zu schaffen. Die an vielen Stellen des Romans als Märchen ausgegebene Welt $(5,306,313)$ erfüllt „einen gewissen pädagogischen Zweck" (5), die für die Phantasie eines. Menschen „keine reale, jedoch eine symbolische Bedeutung" (5) hat, es ist eine utopische Vorankündigung der zukünftigen Welt.

Die große Chance, die Leitfäden der Weltentwicklung selbst in die Hand zu nehmen, sieht Simmel in der Erziehung der jungen Generation: nach bestimmten Prinzipien geformt, werden die heutigen Kinder die Rolle des "Wundertäters" übernehmen und Mamoulians-Häuser zu bauen beginnen.

Das Modell einer solchen Erziehung präsentiert Simmel am Ende des Buches. Am 15. September geht Ruth, Josephines Tochter, zum erstenmal zur Schule: „Die Kinder bestimmten ihre zukünftigen Freunde danach, ob sie ihnen gefielen, und ihre Eltern bestimmten sie danach, ob ihre Eltern ihnen gefielen" (284). Dieser vereinfachten aber zum Teil triftigen Gegenüberstellung Kinder-Erwachsene folgt die paradigmatische Rede eines Lehrers vor den versammelten Müttern, wo es u. a. heißt: „Euren Kindern wird es sehr gleichgültig sein, ob ihre Väter reich oder arm, berühmt oder unbekannt, häßlich oder schön sind - wenn es euch, den Müttern, nicht allzu wichtig ist. Daran müßt ihr denken: es ist nämlich nicht immer ein besonderes persönliches Verdienst, viel Geld zu haben, und es ist noch keine Schande, arm zu sein. Wie es umgekehrt auch noch nicht das Zeichen eines schlechten Charakters ist, reich zu sein, und wie man auch noch nicht deshalb schon ein guter Mensch ist, bloß̧ weil man kein Geld hat ${ }^{25}$. Ihr müßt daran denken, daß eure Kinder die Erwachsenen von morgen sein werden! Und

26. 25 Im Gegensatz zu dieser "Liphre" sind im Roman die Armen a priori gut und ehrlich: Arm sein bedeutet gut sein. "Unsere Menschen" - behauptet einer der Helden \$haben ein gutes Herz und eine große Seele. Sie lachen und weinen und arbeiten. Sie singen Lieder und sind arm"' (240). 
wenn dann die Rechtsanwälte, die Bankdirektoren, die Staatsbeamten und die berühmten Ärzte sagen: Die Arbeiter - das waren doch die Spielgefährten unserer Kindheti! Dann haben wir alle Berechtigung zu hoffen, daß wir einer besseren Welt entgegengehen" (287).

Wenn die Erziehung der Kinder so wirksam sein konnte, wie die Rede des Lehrers, wäre wirklich zu hoffen, daB in ein paar Jahrzehnten Paradies auf Erden aufkommen würde: Von den Worten des Lehrers beeindruckt, gingen die versammelten Mütter noch lange nicht nach Hause: „Sie saßen in den kleinen grünen Bänken und unterhielten sich miteinander. Die Frau des Gaskassierers mit der Frau des Röntgenspezialisten. Und die Frau des Oberstaatsanwaltes mit der Gemüsefrau" (287).

Dieser wieder ins Utopische und in Wunschvorstellungen gesteigerte Vorschlag, durch Erziehung die Antagonismen in der Klassengesellschaft zu beseitigen, wird jedoch schon an der Realität gemessen: es ist eine reale, allerdings komplizierte und schwer realisierbare Möglichkeit, sich in der Zukunft nicht des Wunders bedienen zu müssen. Wenn jedoch die Wirksamkeit dieser Methode am Beispiel der Mütter manifestiert werden sollte, so ist zu befürchten, daß hier versucht wird, Wúnder durch Wundermittel zu' ersetzen. Die Frage nach der Herkunft solcher Lehrkräfte wie der besagte Lehrer eine war, wird hier allerdings nicht gestellt.

Zum Teil nur hat Albrecht Weber Recht, wenn er behauptet, daß Das geheime Brot eigentlich kein "Simmel" ist ${ }^{26}$. Es fehlt hier zwar im Gegensatz sowohl zum ersten Roman wie auch zu allen späteren Sensation, Mord (der einzige Roman Simmels ohne einen einzigen Toten) und das Nebeneinander verschiedener genretypischen Strängen des Unterhaltungsromans (Kriegs-, Kriminal-, Liebes-, Glaubens- und kritischer Zeitroman), doch gewissermaßen am Rande tauchen Problemenkreise auf, die in den meisten späteren Romanen immer wieder variiert werden und den ,eigentlichen" Simmel spüren lassen: das vernachlässigte, im Internat erzogene Kind ${ }^{27}$, die um ihre und ihres Kindes Existenz bangende und sich verkaufende Frau, Geld-Abhängigkeit der Frauen, Faschismus und seine Folgen, reine Liebe, Verhältnis von Gott-Zufall-Mensch usw. usf. Was hier wirklich „fehlt", ist die „Verpackung", die erst den von Millionen gelesenen Simmel ausmachte.

Ohne die „Verpackung" macht sich die konzeptionell-geschichtliche Unbeholfenheit des Autors und/oder seiner Figuren besonders deutlich. Simmels frühe Romane bezeugen die weltanschauliche Verkrampftheit der deutschen Gesellschaft der ersten Nachkriegsjahre und die weltanschauliche Ratlosigkeit des Schriftstellers selbst.

26 Vgl. A. Weber, Das Phänomen Simmel..., a.a.O., S. 112.

${ }^{27}$ Josephine verkauft sich, um ihrer Tochter ihr eigenes - für „Simmel-Kinder" typisches - Los zu ersparen (115-117). 
Die zweifelhaften historiosophischen Konzeptionen, die dem ersten Roman von Simmel zugrunde liegen, und mal auf Entlarvung des (falschen) Geschichtsbewußtseins der deutschen Gesellschaft durch den Autor, mal auf die Bloßstellung seines eigenen fragwürdigen Geschichtsbewußtseins hinweisen, werden im zweiten Roman zusätzlich mit den (noch?) im Dunkeln tappenden, utopisch-naiven Konzeptionen der weiteren geschichtlichen Entwicklung Deutschlands ,ersetzt”. 



\section{INFORMATION ON THE SALE OF}

\section{ADAM MICKIEWICZ UNIVERSITY PRESS PUBLICATIONS}

All Adam Mickiewicz University Press publications are sold by the University Bookshop (Księgarnia Uniwersytecka) 60-813 Poznań, Zwierzyniecka 7. Books published by AMU Press are also available in bookshops of scientific publications all over the country.

Foreign customers can contact directly Adam Mickiewicz University Press, 61-734 Poznań, Nowowiejskiego 55, Poland, telephone 527-380, telex 413260 UAM PL. There they can obtain information on other kinds of transactions and editorial cooperation with AMU Press. 
